\title{
Scattered radiation in DBT geometries with flexible breast compression paddles: A Monte Carlo simulation study
}

\author{
Oliver Diaz, Eloy García, Arnau Oliver, Joan Martí, Robert Martía \\ ${ }^{a}$ VICOROB research institute, University of Girona, Girona, Spain
}

\begin{abstract}
Scattered radiation is an undesired signal largely present in most digital breast tomosynthesis (DBT) projection images as no physically rejection methods, i.e. anti-scatter grids, are regularly employed, in contrast to fullfield digital mammography. This scatter signal might reduce the visibility of small objects in the image, and potentially affect the detection of small breast lesions. Thus accurate scatter models are needed to minimise the scattered radiation signal via post-processing algorithms. All prior work on scattered radiation estimation has used a rigid breast compression paddle (RP) and reported large contribution of scatter signal from RP in the detector. However, in this work, flexible paddles (FPs) tilting from $0^{\circ}$ to $10^{\circ}$ will be studied using Monte Carlo simulations to analyse if the scatter distribution differs from RP geometries. After reproducing the Hologic Selenia Dimensions geometry (narrow angle) with two (homogeneous and heterogeneous) compressed breast phantoms, results illustrate that the scatter distribution recorded at the detector varies up to $22 \%$ between RP and FP geometries (depending on the location), mainly due to the decrease in thickness of the breast observed for FP. However, the relative contribution from the paddle itself (3-12\% of the total scatter) remains approximately unchanged for both setups and their magnitude depends on the distance to the breast edge.
\end{abstract}

Keywords: Scattered radiation, SPR, Monte Carlo simulations, DBT, compression paddle, breast imaging, particle interaction

\section{INTRODUCTION}

Breast compression is a necessary step during mammography procedures to minimise patient movement, tissue cluttering and the effective breast thickness. The latter helps reducing the scattered radiation (which degrades the image contrast and signal to noise ratio), decreases the dose absorbed by the patient and improves the geometric unsharpness.

Traditionally, a rigid compression paddle (RP) is used during image acquisition. However, in the last years, several manufacturers have proposed a compression mode for regular image acquisition using flexible breast compression paddles (FP), where the paddle tilts downward the nipple as the breast is compressed (Fig. 1). Although a previous study discouraged the use of FP as it pushes dense tissue towards the chest wall, ${ }^{1}$ FPs are regularly employed in a large amount of clinics to provide more uniform compression from nipple to chest wall. Manufacturers claim that this minimise the pain and discomfort of the woman during compression.

Prior studies using FPs have investigated image quality and patients discomfort ${ }^{1}$ as previously mentioned, volumetric breast density ${ }^{2}$ or even paddle's tilt estimation. ${ }^{3}$ However, to author's knowledge, no study has been undertaken to analyse the scattered radiation distribution due to FPs. This might be relevant since scattered radiation generated from the compression paddle and breast support plate can contribute up to $31 \%$ of the total scattered radiation observed in digital breast tomosynthesis (DBT) detector. ${ }^{4}$ Therefore, this study investigates how tilted compression paddles influence the distribution of scattered photons recorded within the image receptor. This new knowledge may be important in the design of computer-based scattered radiation reduction tools since more accurate scatter models can be developed.

Further author information: (Send correspondence to Oliver Diaz)

Oliver Diaz: E-mail: oliver.diaz@udg.edu 


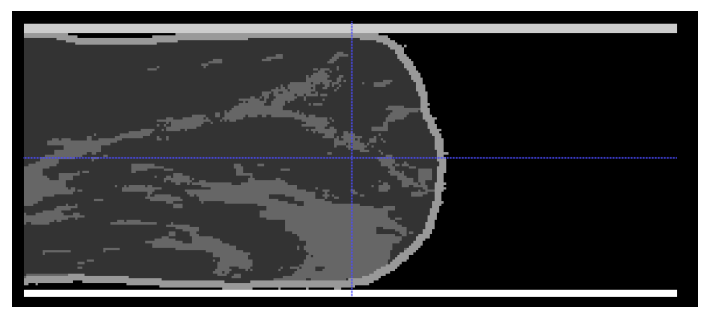

(a) Rigid paddle

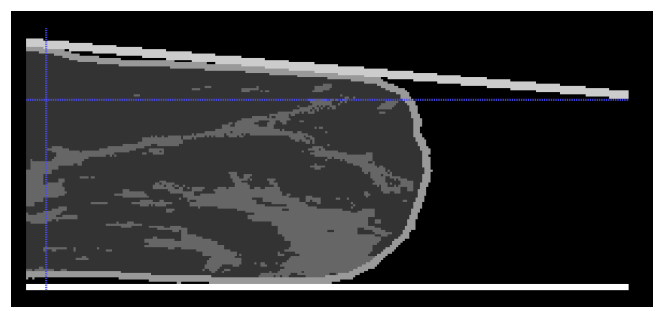

(b) Flexible paddle

Figure 1. Cross section of a $55 \mathrm{~mm}$ thick anthropomorphic breast phantom (at chest wall side) compressed with with a (a) rigid and (b) flexible compression paddle ( $5^{\circ}$ tilt). The latter has been produced with a newly developed in-house breast compression algorithm, based on Ref. 5 .

\section{MATERIALS AND METHODOLOGY}

This section describes the DBT geometry used in the simulations, the breast phantoms investigated as well as the method used to assess the scattered radiation distribution within the image receptor.

\subsection{Digital breast tomosynthesis simulation}

Monte Carlo (MC) simulations, based on the Geant4 toolkit ${ }^{6,7}$ (version 10.01.02), were used in this work to analyse how scattered radiation varies as the compression paddle tilts, simulating FP geometry observed in DBT setups. This MC code was previously validated against the American Association of Physicists in Medicine (AAPM) Task-Group 195 report $^{8,9}$, illustrating an average discrepancy of less than 1\% (maximum 4\%) for scattered radiation calculation within predefined regions of interest. ${ }^{10}$

The DBT geometry used in this work approximates the commercial Hologic Selenia Dimension. It corresponds, from top to bottom, to an infinitesimal x-ray source located at $700 \mathrm{~mm}$ from the detector surface, a $2.8 \mathrm{~mm}$ thick screening compression paddle $\left(180 \times 240 \mathrm{~mm}^{2}\right)$ made of polycarbonate which tilts between 0 and 10 degrees (as observed experimentally), two breast phantom (which are described below), a 1 mm thick breast support plate of carbon fiber matching detector dimension and finally a $200 \mu \mathrm{m}$ thick Selenium detector (240 x $290 \mathrm{~mm}^{2}$ ) with its corresponding cover, located $25 \mathrm{~mm}$ below the breast support (see Figure 2).

A cone beam collimated to match detector area was simulated. The input x-ray spectrum corresponds to a $31 \mathrm{kVp} \mathrm{W} / \mathrm{Al}$ (half-value layer of $0.54 \mathrm{~mm} \mathrm{Al}$ ) with a bin width of $0.5 \mathrm{keV}$, obtained from Ref. 11 . Since the scattered radiation is a low varying signal, a pixelated detector with a pixel pitch of $0.5 \mathrm{~mm}^{2}$ (semi cylindrical phantom) and $1 \mathrm{~mm}^{2}$ (anthropomorphic breast phantom) were used. DBT projection angles of 0 and 7.5 degrees were simulated and the rotation point was located $40 \mathrm{~mm}$ above the detector surface. Note that the detector also tilts with projection angle. Sufficient number of histories were simulated to reach an average error lower than $2 \%$ in scatter to primary ratio calculations.

In this work, six geometry combinations were investigated to study the scattered radiation in setups with RP and FP. For DBT projection angles of $0^{\circ}$ and $7.5^{\circ}$, compression paddle was tilted at $0^{\circ}, 5^{\circ}$ and $10^{\circ}$.

\subsection{Breast phantoms}

Two breast phantom geometries were inserted within the above simulation geometries.

Firstly, a half cylindrical cut section of radius $100 \mathrm{~mm}$ was used, representing a $50 \mathrm{~mm}$ thick compressed breast (at chest wall side) of an homogeneous glandularity of $20 \%$ from a mixture of adipose and glandular tissue $^{12}$ (including $2 \mathrm{~mm}$ thick of skin). Sample breast phantoms compressed with rigid and flexible paddles are depicted in Figure 2. Note that for FP geometry (Fig. 2(b)), the thickness of the breast phantom decreases with its radius. In order to match the same volume as RP compression, its radius was increased (Table 1). Therefore, both RP- and FP-compressed breast phantoms have the same breast volume $\left(785,398.2 \mathrm{~mm}^{3}\right)$.

The second test object used was an anthropomorphic breast phantom. Usually, software-based breast models are compressed mathematically to simulate the rigid compression. However, as previously noted by Diaz et al., ${ }^{13}$ 


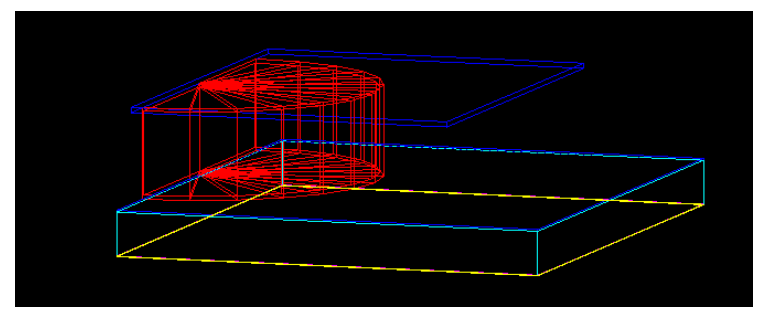

(a) Rigid compression paddle

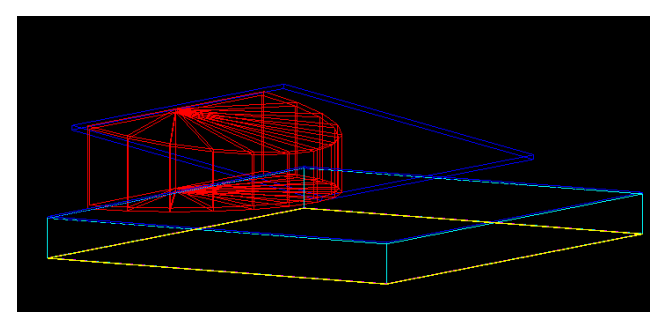

(b) Flexible compression paddle

Figure 2. Semi cylindrical compressed breast model (red) using (a) rigid and (b) flexible compression paddle (blue) after a tilt of $10^{\circ}$. Light blue and yellow corresponds to the support plate and image detector, respectively. Note the decrease in thickness with radius in (b) due to the tilted paddle.

Table 1. Radii used for the semi cylindrical breast phantoms.

\begin{tabular}{|c|c|c|c|}
\hline & Paddle tilt & Phantom radius $\mathbf{( m m})$ & Volume $\mathbf{( m m}^{3} \mathbf{~}$ \\
\hline RP & $0^{\circ}$ & 100.0 & \multirow{2}{*}{$785,398.2$} \\
FP & $5^{\circ}$ & 104.1 & \\
\hline
\end{tabular}

current algorithms for breast compression assume compression between two parallel plates, i.e. compression paddle and breast suppor, representing RP compression geometries. However, the tilt introduced by the FPs provided by several manufacturers have not been previously considered in breast compression algorithms.

The breast model inserted in the MC simulations were generated from three-dimensional (3D) magnetic resonance images (MRI) of a breast, following a methodology similar to Ref 5: the breast region is automatically segmented from background and then, all interest tissues (adipose, glandular and skin) are segmented using intensity based algorithms. In this work, a voxelised breast phantoms is compressed using a compression paddle which tilts several degrees, with respect to its original (parallel-to-detector plane) position (Fig. 1). All compressed breast models have an isotropic voxels of size $1 \mathrm{~mm}^{3}$ and a breast thickness of $55 \mathrm{~mm}$, measured at the chest wall side. Each voxel has an associated label corresponding to skin, adipose or glandular tissue. ${ }^{12}$

\subsection{Scattered radiation calculation}

During MC simulations, all simulated photons generated at the x-ray source were considered primary photons until a coherent (Rayleigh) or incoherent (Compton) scatter interaction occurs, then they were tagged as scattered photons. Simultaneously, the source of scattered photons was identify and recorded. Therefore, the investigated sources of scattered x-ray photons were: (1) air, (2) compression paddle, (3) breast phantom, (4) breast support plate, (5) detector cover as well as (6) x-ray photons which suffer from multiple scattering. When an x-ray photon reaches the detector and deposits energy, via incoherent scattering or photoelectric effect, the deposited energy was stored in the appropriate pixel to create the final (primary or scatter) image projection. In order to reduce noise, the scattered signal was smoothed with a $7 \times 7$ pixels median filter. In this work, the magnitude of scattered radiation observed at the detector was measured in terms of scatter to primary ratio (SPR):

$$
\operatorname{SPR}(x, y)=\frac{P(x, y)}{S(x, y)},
$$

where $S(x, y)$ and $P(x, y)$ correspond to the signal deposited within the $(x, y)$ pixel at the detector, from scattered and primary x-ray photons, respectively. SPR was evaluated across the entire detector, i.e. twodimensional (2D) maps, and within four $10 \times 10 \mathrm{~mm}^{2}$ regions of interest (ROI) at the centre of the image and $20 \mathrm{~mm}$ (ROI 1), $40 \mathrm{~mm}$ (ROI 2), $60 \mathrm{~mm}$ (ROI 3) and $80 \mathrm{~mm}$ (ROI 4) away from chest wall. See black squares in Fig. 3 for the exact location. Scatter profiles were also plotted to investigate scatter sources.

\section{RESULTS}

The colour maps of Fig. 3 represent, for the semi cylindrical phantom, the SPR 2D spatial distributions across the detector from the total scattered radiation. Each row of the figure corresponds to each of the DBT projection 
angles investigated, i.e. $0^{\circ}$ and $7.5^{\circ}$. Since all SPR maps are shown using the same scale $(0-0.8)$, the change in colour observed as the compression paddle is tilted (left to right columns) represents a reduction in the scatter signal as approached near the front edges of the phantom. This effect is mainly led by the reduction in thickness with radius of the phantom (the main scatter source) and the tilted compression paddle, observed when a FP was used (Fig. 2(b)).

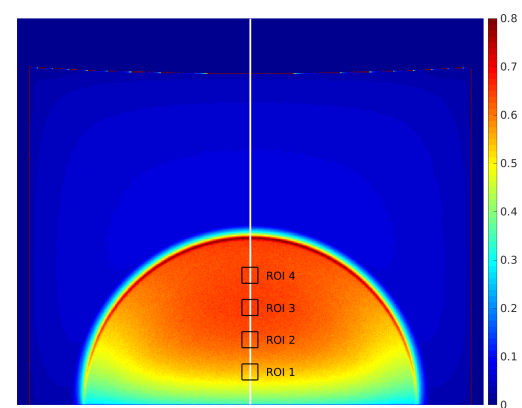

(a) Paddle tilt $0^{\circ}$

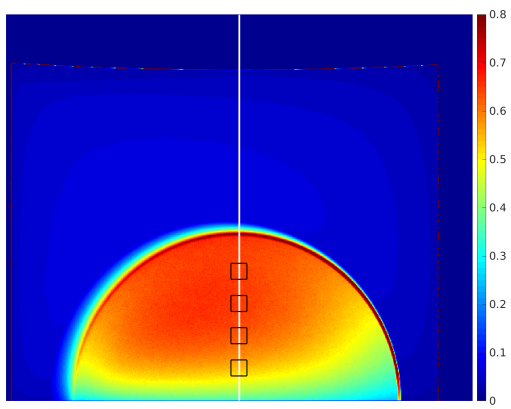

(d) Paddle tilt $0^{\circ}$

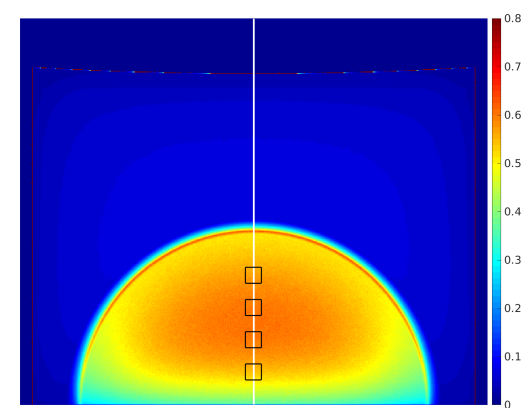

(b) Paddle tilt $5^{\circ}$

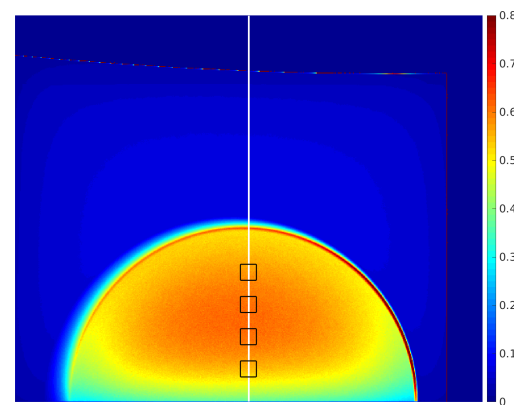

(e) Paddle tilt $5^{\circ}$

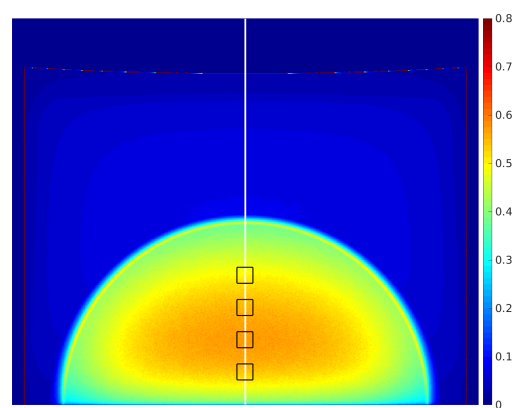

(c) Paddle tilt $10^{\circ}$

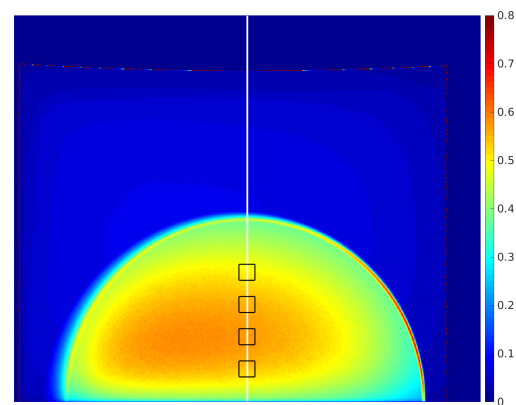

(f) Paddle tilt $10^{\circ}$

Figure 3. SPR maps for different compression paddle tilts using the semi cylindrical phantom. First and second rows correspond to DBT projection at $0^{\circ}$ and $7.5^{\circ}$, respectively. Black squares represent the location of the ROIs and the white vertical line is used to show the profiles depicted in Fig. 4.

This effect is more clearly observed in Fig. 4, where SPR profiles along the vertical white line are plotted for the $0^{\circ}$ DBT projection angle. This time, all scatter sources have been differentiated, showing the total scatter signal as black. The aforementioned thickness reduction of the phantom with its radius and the inclination of the paddle have converted an almost plateau region observed for RP (solid black line), between distances 60 to $100 \mathrm{~mm}$, to a dramatic reduction in scatter at paddle tilt of $10^{\circ}$ (dotted black line). Since the radius of the phantom is increased, there is less penetration of scattered photons from both compression paddle and breast support near the edges of the phantom.

Furthermore, the total average SPR value recorded for each of the previously defined ROIs (ROI 1, ROI 2, ROI 3 and ROI 4) and all DBT geometries studied are shown in Table 2. No significant difference was observed in SPR values between $0^{\circ}$ and $7.5^{\circ}$ projection angles. However, it was noted that SPR values decrease with paddle's tilt for all ROIs, except for ROI 1 which is located close to the chest wall plane $(20 \mathrm{~mm})$. The closer to the edge of the phantom, the larger the SPR reduction. The average SPR reduction for all ROIs was $6 \%$ and $12 \%$ for paddle's tilt of $5^{\circ}$ and $10^{\circ}$, respectively.

Table 3 illustrates the analysis of the sources of the scatter radiation. It was observed that most of the total scattered radiation recorded within the four ROIs came from the phantom (55-58\%) and from multiple interactions (30-31\%). The contribution from the compression paddle and breast support was $6 \%$ and $5-6 \%$, respectively. Finally, the contribution from the detector cover and air were less than $1 \%$ in both cases.

Similarly for the semi circular phantom, Fig. 5 and table 4 illustrate the SPR 2D maps and the average SPR values within each ROI for the anthropomorphic breast phantom, respectively. As shown previously, SPR values 


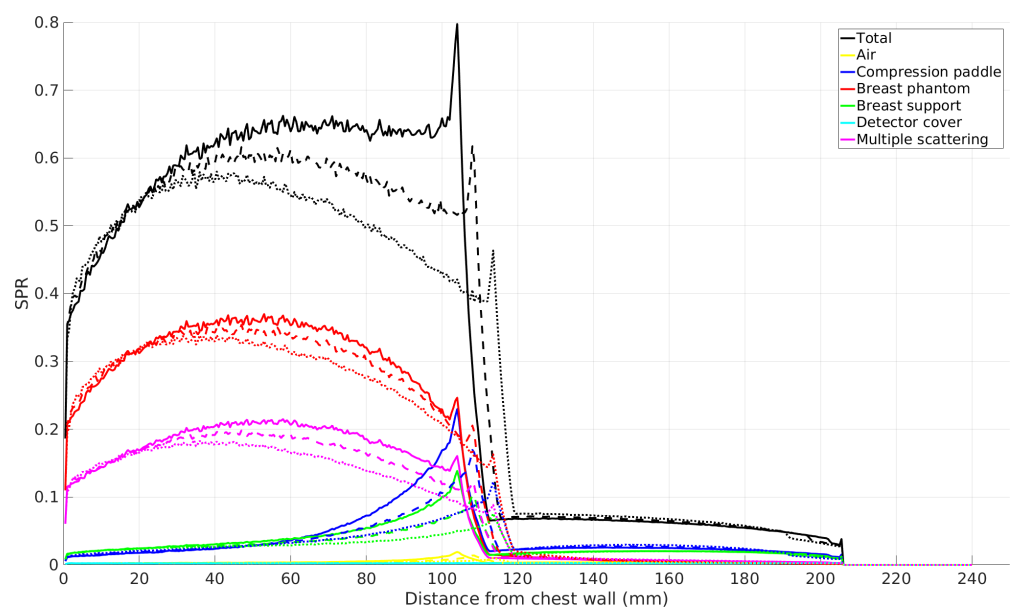

Figure 4. SPR from each scatter source observed for $0^{\circ}$ DBT projection angle. Profiles along white vertical line of Fig. 3. Solid, dashed and dotted line corresponds to paddles tilts of 0,5 and $10^{\circ}$, respectively.

Table 2. SPR values (mean \pm stdev) for the semi cylindrical breast phantom. See Fig. 3 for ROI's location.

\begin{tabular}{|c|c|c|c|c|c|}
\hline DBT projection & Paddle tilt & ROI 1 & ROI 2 & ROI 3 & ROI 4 \\
\hline \multirow{2}{*}{$0^{\circ}$} & $0^{\circ}$ & $0.54 \pm 0.02$ & $0.62 \pm 0.01$ & $0.65 \pm 0.01$ & $0.64 \pm 0.01$ \\
& $5^{\circ}$ & $0.54 \pm 0.01$ & $0.60 \pm 0.01$ & $0.60 \pm 0.01$ & $0.57 \pm 0.01$ \\
& $10^{\circ}$ & $0.54 \pm 0.01$ & $0.57 \pm 0.00$ & $0.55 \pm 0.01$ & $0.50 \pm 0.01$ \\
\hline \multirow{2}{*}{$7.5^{\circ}$} & $0^{\circ}$ & $0.54 \pm 0.02$ & $0.63 \pm 0.01$ & $0.65 \pm 0.01$ & $0.64 \pm 0.01$ \\
& $5^{\circ}$ & $0.54 \pm 0.02$ & $0.60 \pm 0.01$ & $0.61 \pm 0.01$ & $0.57 \pm 0.01$ \\
& $10^{\circ}$ & $0.54 \pm 0.01$ & $0.57 \pm 0.00$ & $0.55 \pm 0.01$ & $0.50 \pm 0.01$ \\
\hline
\end{tabular}

Table 3. Scatter contribution (mean \pm stdev) in percentage from each of the scatter sources studied within all four ROIs.

\begin{tabular}{|c|c|c|c|c|c|c|c|}
\hline DBT projection & Paddle tilt & Air & Paddle & Phantom & Support plate & Detector Cover & Multiple scatter \\
\hline \multirow{2}{*}{$0^{\circ}$} & $0^{\circ}$ & $1 \pm 0$ & $6 \pm 4$ & $55 \pm 4$ & $6 \pm 2$ & $0 \pm 0$ & $31 \pm 2$ \\
& $5^{\circ}$ & $1 \pm 0$ & $6 \pm 3$ & $56 \pm 3$ & $6 \pm 1$ & $0 \pm 0$ \\
\hline \multirow{2}{*}{$0^{\circ}$} & $10^{\circ}$ & $1 \pm 0$ & $6 \pm 2$ & $58 \pm 2$ & $5 \pm 1$ & $0 \pm 0$ \\
& $0^{\circ}$ & $1 \pm 0$ & $6 \pm 4$ & $55 \pm 4$ & $6 \pm 2$ & $0 \pm 0$ \\
& $5^{\circ}$ & $1 \pm 0$ & $6 \pm 3$ & $56 \pm 3$ & $6 \pm 1$ & $0 \pm 0$ \\
& $10^{\circ}$ & $1 \pm 0$ & $6 \pm 2$ & $57 \pm 2$ & $5 \pm 1$ & $31 \pm 2$ \\
\end{tabular}

did not change significantly within same ROI for projection angles of $0^{\circ}$ and $7.5^{\circ}$. However, differences in SPR are observed for regions close to the edge of the phantom (ROI 2, 3 and 4) in similar magnitudes: average SPR decrease of $8 \%$ and $11 \%$ for paddle's tilt of $5^{\circ}$ and $10^{\circ}$, respectively.

Table 4. SPR values (mean \pm stdev) for the realistic breast phantom. See Fig. 5 for ROI's location.

\begin{tabular}{|c|c|c|c|c|c|}
\hline DBT projection & Paddle tilt & ROI 1 & ROI 2 & ROI 3 & ROI 4 \\
\hline \multirow{2}{*}{$0^{\circ}$} & $0^{\circ}$ & $0.57 \pm 0.02$ & $0.64 \pm 0.03$ & $0.62 \pm 0.02$ & $0.70 \pm 0.03$ \\
& $5^{\circ}$ & $0.54 \pm 0.02$ & $0.60 \pm 0.02$ & $0.58 \pm 0.02$ & $0.62 \pm 0.03$ \\
& $10^{\circ}$ & $0.54 \pm 0.02$ & $0.58 \pm 0.02$ & $0.55 \pm 0.02$ & $0.56 \pm 0.03$ \\
\hline \multirow{3}{*}{$7.5^{\circ}$} & $0^{\circ}$ & $0.57 \pm 0.03$ & $0.64 \pm 0.02$ & $0.61 \pm 0.02$ & $0.68 \pm 0.04$ \\
& $5^{\circ}$ & $0.53 \pm 0.02$ & $0.60 \pm 0.01$ & $0.57 \pm 0.02$ & $0.60 \pm 0.04$ \\
& $10^{\circ}$ & $0.54 \pm 0.02$ & $0.58 \pm 0.01$ & $0.54 \pm 0.03$ & $0.54 \pm 0.03$ \\
\hline
\end{tabular}

\section{DISCUSSION AND CONCLUSIONS}

This paper illustrates, for the first time, the effect of the flexible compression paddle on the scattered radiation distribution at the image receptor. MC simulations, using an homogeneous and a more realistic (and heterogeneous) breast phantom, were used. This FP compression mode, which is used regularly in a large amount of breast clinics, might reduce significantly the thickness of the breast in the edges around the nipple. Since the breast represents the major source of scattered radiation, the rapid breast thickness reduction due to the FP compression should be taken into account for software-based scatter reduction tools.

As expected, MC simulations using FP compression illustrated that the major source of scatter radiation comes from the thickest material, i.e. the breast phantom. Its contribution to the total scatter is slightly increased (5\%) with paddle's tilt, at expenses of a small decrease in the contribution from the compression paddle and breast support $(2-3 \%)$. Since the breast thickness decreases with the radius, the penetration of scatter from the 


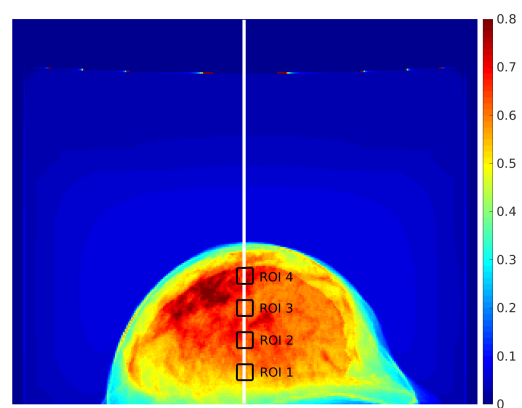

(a) Paddle $0^{\circ}$

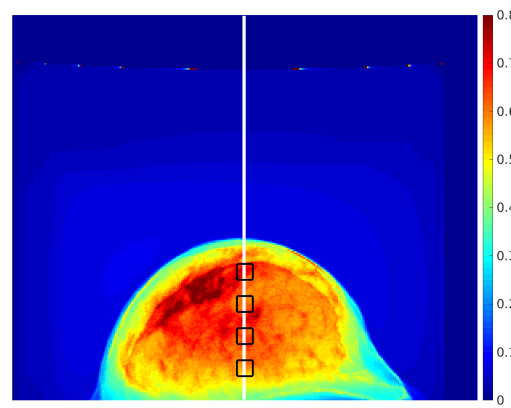

(d) Paddle $0^{\circ}$

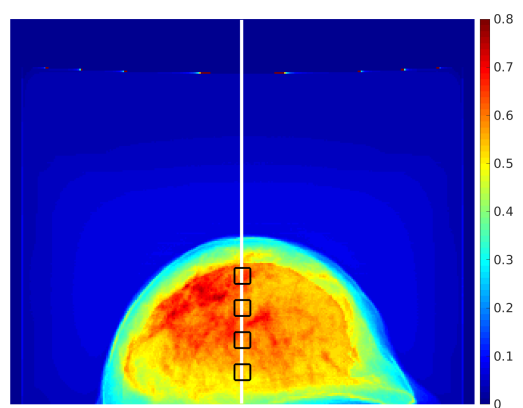

(b) Paddle $5^{\circ}$

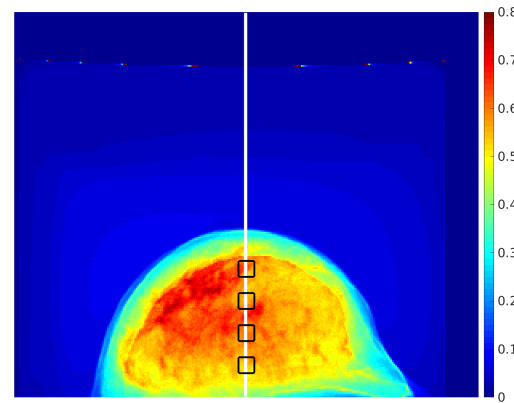

(e) Paddle $5^{\circ}$

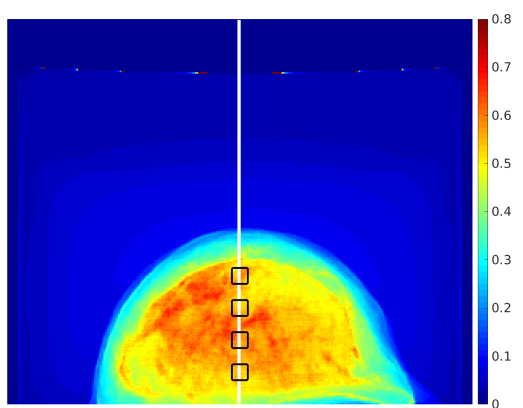

(c) Paddle $10^{\circ}$

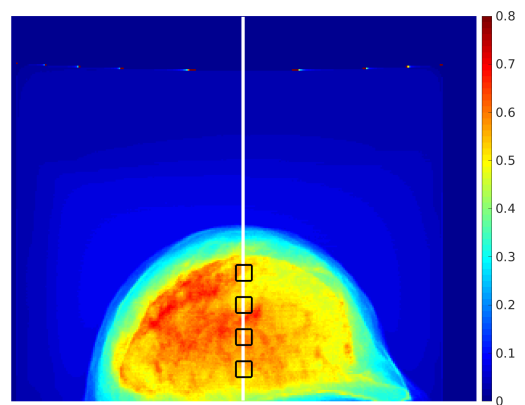

(f) Paddle $10^{\circ}$

Figure 5. SPR maps for different compression paddle tilts using the anthropomorphic breast phantom. First and second rows correspond to DBT projection at $0^{\circ}$ and $7.5^{\circ}$, respectively. Black squares represent the location of the ROIs.

compression paddle differs between RP and FP compression near th edges of the breast. The rest of the scatter sources investigated (i.e. air, detector's cover and multiple scattering) remained unchanged with paddle tilt. In general, it was observed that the distribution of scattered radiation across the detector varies with location in the image.

For the four $10 \times 10 \mathrm{~mm}^{2}$ ROIs investigated (at 20, 40, 60 and $80 \mathrm{~mm}$ from chest wall plane), an average total SPR decrease of $6 \%(1-12 \%)$ was observed for paddle tilt of $5^{\circ}$ and an average decrease of $12 \%(1-22 \%)$ was seen for paddle tilt of $10^{\circ}$. No significant difference was observed between the projection angles of $0^{\circ}$ and $7.5^{\circ}$, as employed by the Hologic Selenia Dimensions systems.

Furthermore, this work used an anthropomorphic breast phantom which included a novel deformation to match real observations in FP geometries. This heterogeneous phantom, generated from clinically acquired MR images of a woman, illustrates a more realistic tissue distribution to analyse the scatter field within the detector. Results illustrate similar behaviour of the scatter than previously observed for the semi cylindrical and homogeneous breast phantom.

Based on the geometry and phantoms investigated in this work, we could conclude that the scatter radiation varies more rapidly with flexible compression paddles than rigid paddles. Despite further investigations are needed to analyse a larger range of breast thicknesses and projection angles (e.g. $25^{\circ}$ ), initial results demonstrate that scatter models generated using RP, can differ greatly for FP, especially near the edges, mainly due to the decrease in breast thickness. Therefore, when developing an accurate scatter reduction algorithm, this should be led by local breast thickness calculations.

\section{ACKNOWLEDGMENTS}

This work is part of the SCARtool project (H2020-MSCA-IF-2014, reference 657875), a research funded by the European Union within the Marie Sklodowska-Curie Innovative Training Networks. Also, some of the authors have been partially supported from the Ministry of Economy and Competitiveness of Spain, under project 
reference DPI2015-68442-R, University of Girona under project reference MPCUdG2016/022, and the FPI grant BES-2013-065314.

\section{REFERENCES}

[1] Broeders, M., ten Voorde, M., Veldkamp, W. H., van Engen, R., van Landsveld-Verhoeven, C., 't Jong Gunneman, M., de Win, J., Greve, K., Paap, E., and den Heeten, G., "Comparison of a flexible versus a rigid breast compression paddle: pain experience, project-ed breast area, radiation dose and technical image quality," European Radiology 25(3), 821-829 (2015).

[2] Kallenber, M., van Gils, C., Lokate, M., den Heeten, G., and Karssemeijer, N., "Effect of compression paddle tilt correction on volumetric breast density estimation," Physics in Medicine and Biology 57(16), 5155-5168 (2012).

[3] Kallenber, M. and Karssemeijer, N., "Compression paddle tilt correction in full-field digital mammography," Physics in Medicine and Biology 57(3), 703-715 (2012).

[4] Sechopoulos, I., Suryanarayanan, S., Vedantham, S., D'Orsi, C., and Karellas, A., "Scatter radiation in digital tomosynthesis of the breast," Medical Physics 34(2), 564-576 (2007).

[5] Garcia, E., Oliver, A., Diez, Y., Diaz, O., Georgii, J., Gubern-Merida, A., Marti, J., and Marti, R., "Comparing regional breast density using full-field mammograms and magnetic resonance imag-ing: A preliminary study," in [Proceeding of the 3rd MICCAI Workshop on Breast Image Analysis], 9699, 33-40, Springer International Publishing (2015).

[6] Agostinelli, S., Allison, J., Amako, K., Apostolakis, J., Araujo, H., Arce, P., Asai, M., Axen, D., Banerjee, S., and Barrand, G., "Geant4 - a simulation toolkit," Nuclear Instruments and Methods in Physics Research 506(3), 250-303 (2003).

[7] Allison, J., Amako, K., Apostolakis, J., Araujo, H., Arce, P., Asai, M., Barrand, G., Capra, R., and Chauvie, S., "Geant4 development and applications," IEEE Transactions on Nuclear Science 53(1), 270-278 (2006).

[8] Sechopoulos, I., Ali, E., Badal, A., Badano, A., Boone, J., Kyprianou, I., Mainegra-Hing, E., McMillan, K., McNitt-Gray, M., Rogers, D., Samei, E., and Turner, A., "Monte carlo reference data sets for imaging research: Executive summary of the report of aapm research committee task group 195," Medical Physics 42(10), 5679-5691 (2015).

[9] Sechopoulos, I., Ali, E., Badal, A., Badano, A., Boone, J., Kyprianou, I., Mainegra-Hing, E., McMillan, K., McNitt-Gray, M., Rogers, D., Samei, E., and Turner, A., "The report of aapm task group 195: Monte carlo reference data sets for imaging research."

[10] Marimon, E., Nait-Charif, H., Khan, A., Marsden, P., and Diaz, O., "Detailed analysis of scattered contribution from different simulated geometry of x-ray detectors," in [Breast Imaging: 13th International Workshop, IWDM 2016, Malmö, Sweden, June 19-22, 2016, Proceedings], 9699, 203-210, Springer International Publishing (2016).

[11] Boone, J., Fewell, T., and Jennings, J., "Molybdenum, rhodium and tungsten anode spectral models using interpolating polynomials with applications to mammography," Medical Physics 24(2), 1863-1874 (1997).

[12] Hammerstein, G., Miller, D., White, D., Masterson, M., Woodard, H., and Laughlin, J., "Absorbed radiation dose in mammography," Radiology 130, 485-491 (1979).

[13] Diaz, O., Oliver, A., Ganau, S., Garcia, E., Marti, J., Sentis, M., and Marti, R., "Feasibility of depth sensors to study breast deformation during mammography procedures," in [Breast Imaging: 13th International Workshop, IWDM 2016, Malmö, Sweden, June 19-22, 2016, Proceedings], -, Springer International Publishing (2016). 